\section{A PLEA FOR MORE RADICAL OPERATIONS IN CANCER OF THE LIPS AND TONGUE.*}

\section{N. EISENDRATH, M.D. CHICAGO.}

The decreased number of recurrences after a thorough removal of the breast according to the method of Halsted and others has taught us that the same principles could be applied in the surgical treatment of carcinomata elsewhere in the body. In no class of cases does this seem more promising than in carcinomata of the more superficial character like those of the face, tongue, lips, ear, etc.

MODE OF GROWTH OF SQUAMOUS-CELLED CARCINOMATA.

Carcinomata of the lips, tongue, ear, etc., all belong to the flat or squamous-celled type. They spread almost exclusively either by continuity of tissue or along the lymphatic spaces and vessels to the lymph nodes which normally drain the region involved. $\mathrm{Me}-$ tastatic growths in the internal viscera or long bones are very rare in the squamous - celled type of carcinoma.

von Winiwarter and Landau were unable to find internal metastases in a large number of cases examined by them. Küttner ${ }^{1}$ found only four in which there were such deposits in a number of cases of cancer of the tongue reported from nine German university clinics.

These data confirm the frequently observed clinical fact that recurrence of carcinoma of the lips, tongue, etc., usually takes place close to the original seat of operation or in the adjacent lymph nodes. Such a local or regional recurrence usually manifests itself from one to three years after operation; but cases have median line; $c$, submaxillary lymph node; $d$, sympl nodes in geniohyold mus they did not appear for eiglit or ten years. The latter are a rare exception.

There are but few reliable statistics which will aid us in judging of the percentage of permanent recovery after the older and less radical operations for removal of the class of carcinomata of which this paper treats. The chief reason for this is the great difficulty in following the cases for a long period. Küttner was able to collect 162 cases from nine hospitals in which the non-

\footnotetext{
* Read at the annual meeting of the Illinois State Medical So cletr, May 16,1906 .

1. Kittner: Beitr., z. KIln. Chir., vol. xxi.
}

radical operation had been performed for cancer of the tongue and found only 13 per cent. of permanent recoveries.

There is no form of carcinoma in which involvement of the lymph nodes draining the area involved takes place as early as in carcinoma of the tongue, lips, etc. It is for this reason that operations for the radical removal of all infected territory seem to offer such favorable opportunities for permanent cures.

LYMPH NODES WHICH DRAIN THE LIPS, TONGUE, FACE AND EARS.

These have been carefully investigated by Sappey, Küttner, ${ }^{1}$ Polya, ${ }^{2}$ Poirier ${ }^{3}$ and others and can be best understood by a reference to Figures 1,2 and 3 . They are as follows:

1. For the Tongue (Fig. 1).-Submaxillary lymph nodes receive lymph from the edge, lower surface and middle of dorsum of tongue and entire floor of mouth. Superior and inferior deep cervical nodes drain entire tongue and floor of mouth. In addition to these there are s o m e intramuscular nodes situated in the geniohyoid muscles, and at times retrograde lymph currents may involve the parotid nodes. There is much intercommunication of lymphatics so that a carcinoma involving only one side of the tongue may invade the lymph nodes of both sides of the neck. The most important node is situated at the bifurcation of the common carotid arteries. This node lies deep beneath the posterior belly of the digastric muscle. In order thoroughly to remove the deep nodes which drain the tongue, it is also necessary to excise the lower part of the parotid salivary gland.

2. For the Lips (Fig. 2.)-The chief lymph nodes which drain the lips are situated in the submental and submaxillary regions. Occasionally infection of one of the deep cervical nodes will take place at an early period. The submental lymph nodes draining the lips are situated just beneath the skin between the two anterior bellies of the digastric muscles. The submaxillary nodes lie embedded in the subcutaneous fat of this region in close relation to the capsule of the submaxillary salivary

2. Polya: Deutsch. Ztschr. f. Chir., vol. Ixv.

3. Poirler, Cuneo and Lamere: "The Lymphatics." 
gland. They are at times found within the latter so that it is always safer to remove the submaxillary salivary gland itself.

Involvement of the deep cervical nodes does not take place until a later period of the primary growth, but in a radical operation, even at an early stage, the possibility of their involvement must be borne in mind.

3. For the Ear (Fig. 3).-The lymph nodes draining the external ear are chiefly the deep cervical, although at times the parotid and mastoid nodes may be first affected.

4. For the Nose, Face and Cheeks.-The lymphatic drainage of the nose, face and cheeks is chiefly into the submental and submaxillary nodes and from these into the deep cervical at a comparatively late period of the development of the primary growth. The lymph from the lateral portion of the face drains into the parotid nodes situated on, within and beneath the capsule of the parotid salivary gland.

One can not in general rely on any one set of nodes being affected, but must remove all of them in order to

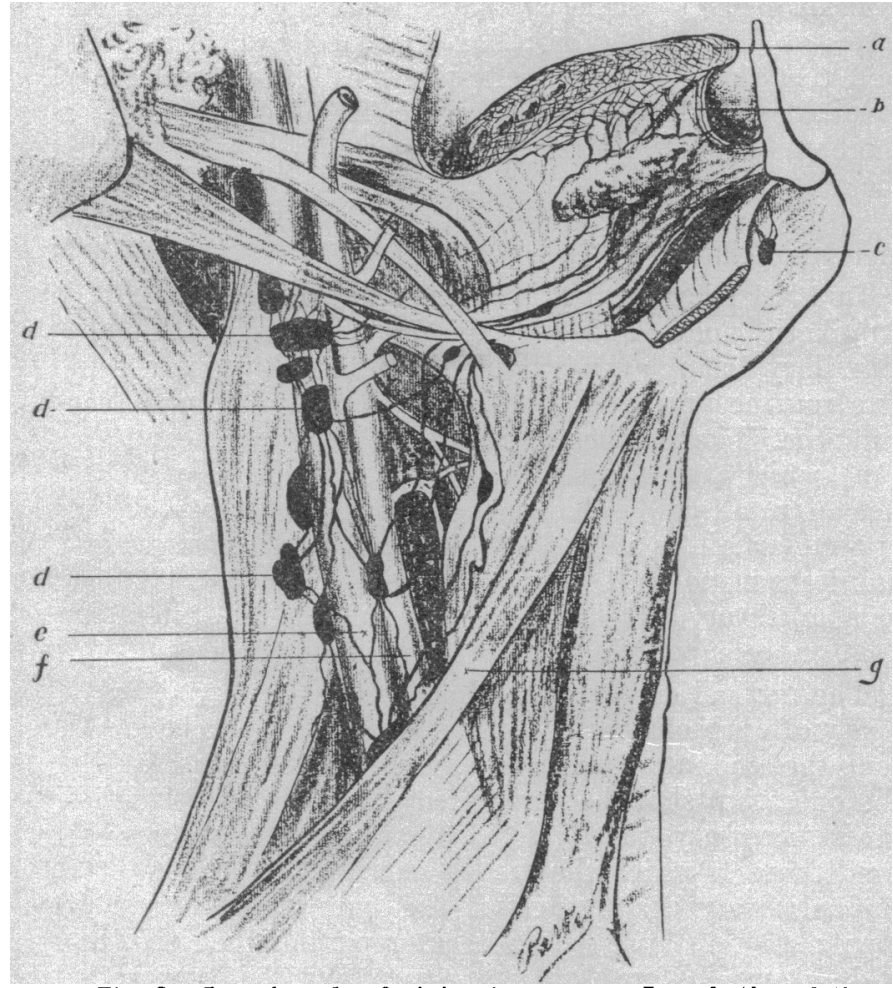

Fig. 2.-Lymph nodes diaining tongue: $a$, Lymphatics of tip of the tongue; $b$, lymphatic trunks of sides of tongue and floor of mouth, draining into $c$, submaxillary and submental nodes, and into $d$, deep cervical nodes; $e$, internal jugular vein; $f$, common carotid artery $g$, omohyoid muscle.

be thorough. One should not depend on external palpation for enlarged lymph nodes before undertaking a radical operation. Such enlargement may be simply inflammatory or there may be a number of deep nodes which are not palpable.

IIISTORY OF OPERATION.

Before beginning the description of the methods I will refer briefly to the development of this tendency toward more radical operations in this class of carcinomata.

The majority of the articles have appeared during the past eight years. The first surgeon to advocate thorough removal of all lymphatic nodes was Butlin.4 In a paper

4. Butlin: Brit. Med. Jour., vol. 1, 1898, and vol. 1, 1905. published in 1898, he stated that he had employed the method since $\mathbf{1 8 9 5}$, in a more or less systematic manner, for cancer of the tongue. Since 1898 a number of articles have appeared advocating the same procedure for cancer of the tongue, lips, etc. Among these may be mentioned those of Crile, Küttner, ${ }^{1}$ Polya, ${ }^{2}$ Stewart, Cheyne, ${ }^{6}$. Stieda, Ebel, ${ }^{8}$ Boyd and Unwin $^{9}$ and Whitehead. ${ }^{10}$



Fig. 3.- Lymph nodes draining cheeks and lips: $a$, Lymph nodes of cheek; $h$, lymph nodes of lower lip and chin region draining into submaxiliary and submental glands; $c$, deep cervical nodes.

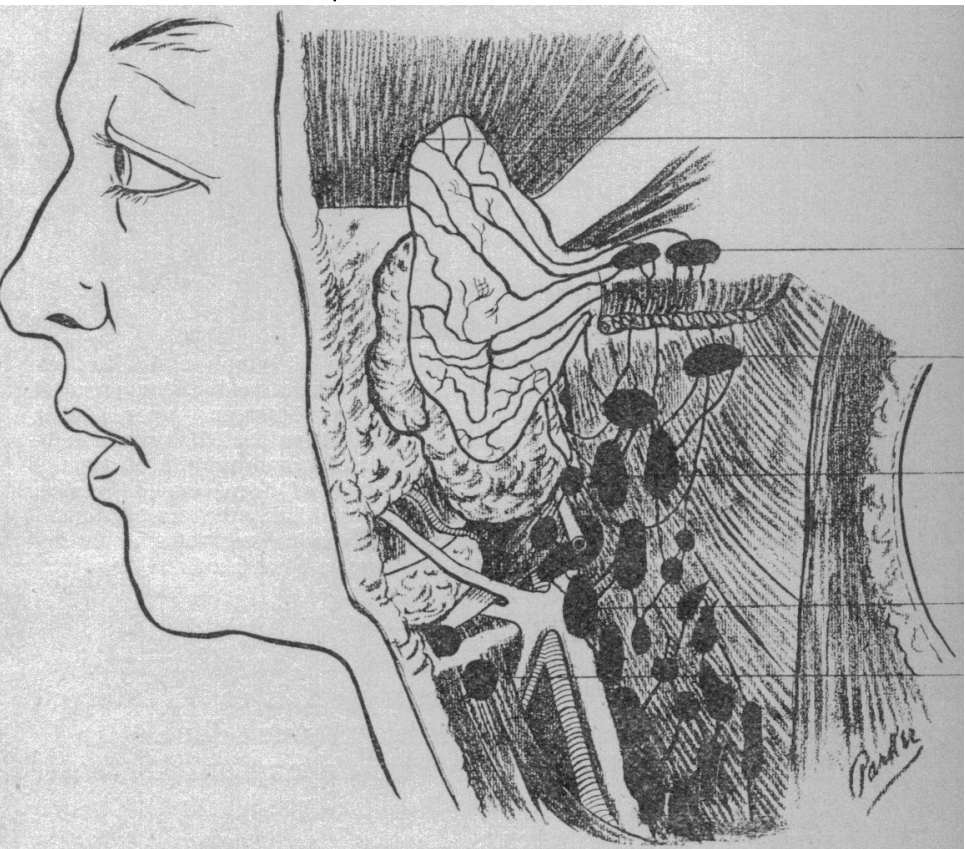

Fig. 4.-Lymph nodes draining ear (Poirier); $a$, Lymphatic trunks of auricle; $b$, mastoid, $c, d, e$, and $t$, deep cervical nodes.

Unfortunately there are but few statistics up to the present time which will enable one to form an opinion as to what extent these more radical operations have less-

5. J. C. Stewart: St. Paul Med. Jour., vol. vi, 1904

6. Cheyne : Practitioner, April, 1899.

7. Stieda: Archiv. f. Klin. Chir., vol. Ixiil.

8. Ebel: Beitr. z. Klin. Chir., vol, xl.

9. Boyd and Inwin : Practitioner, May, 1903.

10. Whitehead, W.: Practitloner, May, 1903. 
ened the percentage of recurrence. As in the case of the development of the same mode of operation in cancers of the breast, it will be necessary to collect a large number of cases and await a considerable time after operation before we can judge of the value of the same principle as applied to cancers of the lips, tongue, etc. It would seem as though a period of at least three years should elapse before results could be fairly judged.

Butlin ${ }^{4}$ began to perform the operation for the complete removal of the contents of the anterior triangle of the neck in December, 1895, or January, 1896. Between that period and December, 1901, there were 28 of these operations, and, in a paper published in 1905, he says: "I will merely take the number of persons who died of affection of the gland without recurrence in the mouth and the number of successful cases, that is, of persons who were alive and well three or more years after the operation, or who died of some other disease than cancer more than three years after the operation. There were 28 cases of removal of the contents of the anterior triangle of the neck from January, 1896, to December, $1901 ; 4$ patients died of affection of glands without recurrence in the mouth; successful cases, 10.

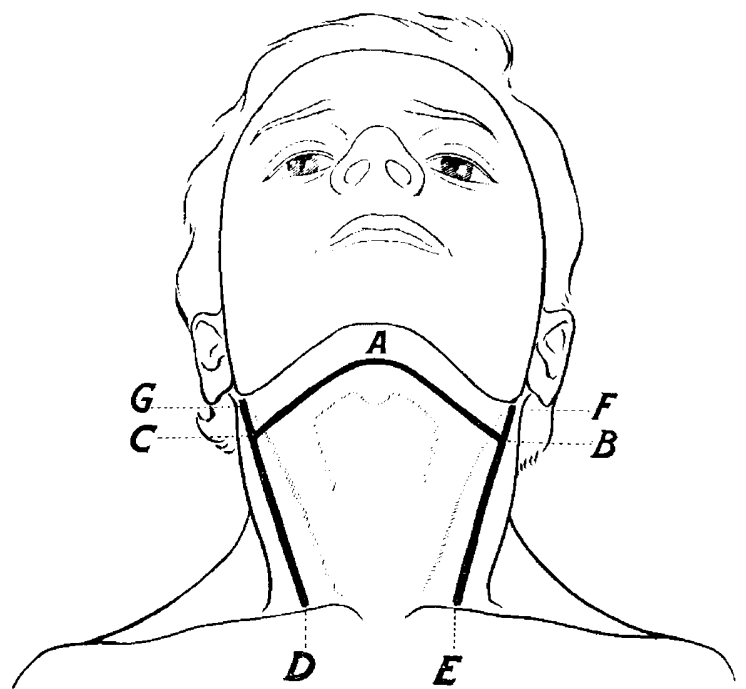

Fig. 5.-Incision employed in extirpation of lymph nodes in car cinoma of the lips, tongue, etc. C, A, B, transverse incision paralle to lower bolder of lower jaw, and midway between upper border of thyroid and lower jaw for extirpation of submaxillary and submental glands; $G, C, D$ and $F, B, F$, incisions over middle of sternocleidomastoid muscles to permit extirpation of deep cervical zodes, with or without the renoval of the sternocleidomastold and interna jugular vein. The nodes in the supraclavicular rerion may be removed by an incision parallel to the upper border of the clavicle, carried outwards from $w$ or $\mathbf{E}$ respectively.

Compare with these the results of the old operation, in which the glands which could be felt to be enlarged were taken out, but no more than this was done. Up to January, 1896, I performed this operation 31 times. Of these 31 cases of removal of enlarged glands before January, 1896,8 patients died of affection of glands without recurrence in the mouth; successful cases, 5 .

Again, take another series of cases to compare with the first series. Between January, 1896, and December, 1901 , Butlin operated on 34 patients for the removal of cancer of the tongue without removal of the glands of the neck. Of these 34 cases 9 patients died of affection of the glands without recurrence in the mouth; successful ones. 9."

It will be scen at a glance that by far the most successful results are those which were procured by the removal of the contents of the anterior triangle. But the mere statement of numbers does not tell anything. Allowance has been made for certain conditions which were present or which were absent in each of these three series of cases. In the first series of cases, there were 18 patients in whom the glands were perceptible to touch at the time they were removed, $\gamma$ in whom they were not perceptible, and 3 of whom there was no note on this important point. By far the best results were obtained in the 7 cases in which the glands were not perceptible to touch For 4 of these patients are among the 10 successful cases. Of course, in the second series the glands werc perceptible in every one of the 31 patients, and that was why they were removed. So far from thinking the results bad, he was surprised to find they were so good. For it is a remarkable circumstance that five persons, under such conditions, should have been cured by so incomplete an operation. In more than 1 of these 5 cases the glands were proved by microscopic examination to contain epithelioma. Yet one of these patients was alive and well nearly twenty years after the operation.

Probably no corrections are required in the third series of 34 cases. For if, on the one hand, it may be said that these 34 cases comprise the lightest and most favorable of all the cases (which is, of course, true), there were, on the other hand, some of them in which the glands were not removed, because they were beyond reach of removal, and the operation on the tongue was merely palliative.

In 1903 I began to employ the radical removal of the lymphatic nodes and surrounding tissues in the submaxillary triangle and along the internal jugular vein as a routine procedure in cancer of the lips, tongue, car and face. The number of cases is still too small and the time which has elapsed too short to render a publication of results advisable at the present time, so that the present article is intended more as a preliminary communication to call the attention of the profession to a much-needed change in our surgical treatment of these more superficially situated carcinomata.

The method employed at the present time by the majority of surgeons varies chiefly, $a$, as to the incision; $b$, as to the amount of tissue to be removed; $c$, as to. whether the operation shall be performed at one or two sittings. It is necessary to divide the cases into two classes: first, those in which the case is seen by the surgeon at a comparatively carly stage of the lymph node involvement; second, those seen at a later period when the secondary growths are very extensive. For the former the first method of operation to be described is to be employed; for the more advanced cases the methods recommended by Crile, Butlin ${ }^{4}$ and others are to be used.

\section{TECHNIC FOR LIESS ADVANCED CASES.}

1. Removal of Primary Growth.-In cancers of the lips and tongue the amount to be removed varies, of course, with the extent of the lesion. As a general rule, one should never be content with excising at least a half inch of healthy tissue on each side of the growth. In cancer of the tongue which extends beyond the median line, it is better to remove the entire organ, since the lymphatics of both sides of the neck are probably involved. Whether the tongue shall be removed, $a$, through the mouth after the method of Whitehead; or, $b$, through an incision parallel to the lower jaw, i. e., from below, combined with temporary resection of the jaw, is a matter of individual preference. The majority of surgeons both in this country and abroad prefer the Kocher 
method, i. e., from below the jaw. It can be readily combined with radical removal of infected lymphatic tissue, or combined with ligation of the external carotid artery to be referred to below.

Temporary resection of the lower jaw in the median line after the method of Langenbeck adds greatly to the (xposure of the operative field.

2. Removal of Infected Lymphatic Nodes.-In the majority of cases cancer of the lips, tongue, ear, face and cheek, the incisions shown in Figures 5 and 7 will suffice. 1 have used them since 1903 and they give excellent access to every part of the neck. In cases of cancer of the lips only the first portion need be employed; viz., C, A, B (Fig. 5). This consists of an incision through the skin and subcutaneous fat extending parallel to the lower jaw and midway between the lower border of the latter and the upper border of the thyroid cartilage from the sternocleidomastoid muscle on one side of the neck to the same structure on the opposite side.

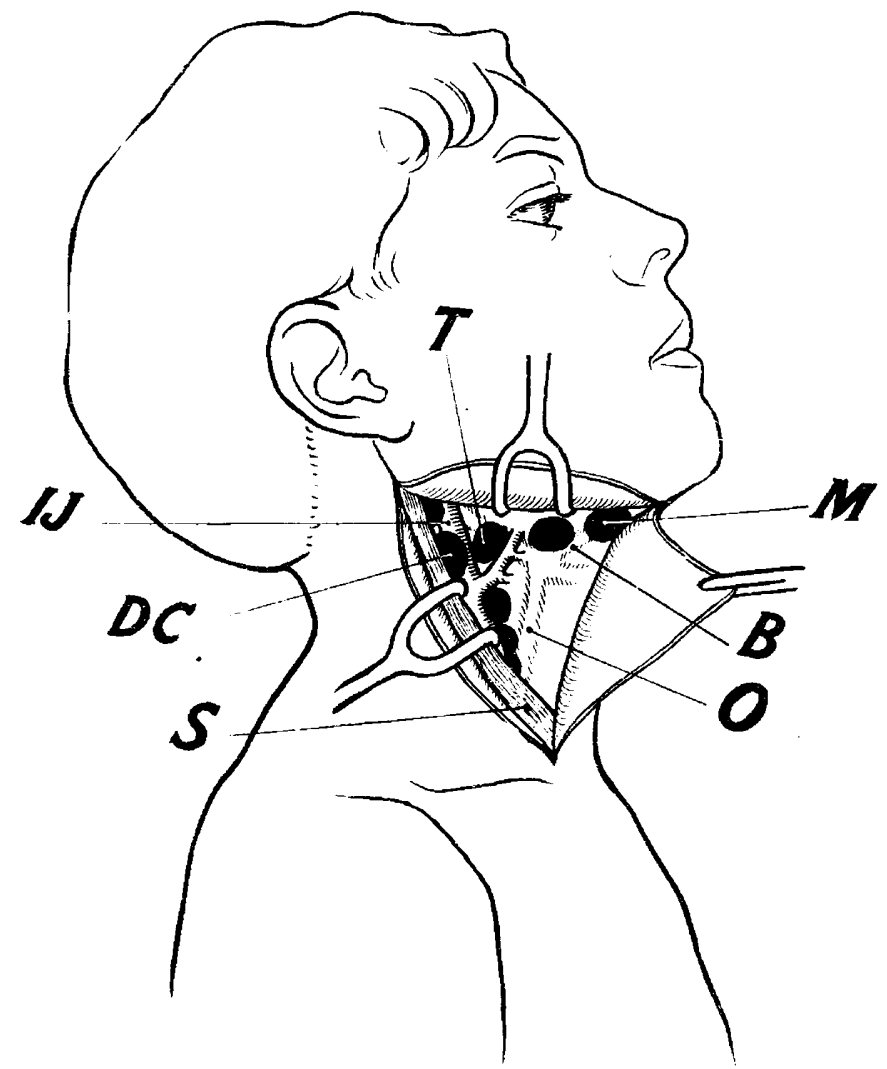

lis. t.- Isocation of most frequentiv affected lymple nodes in calcinoma of the tongue: $\mathbf{I}$, submaxillary nodes; $T$, deep cervical node lying between internal and anterior jugular veins; $D$, C, deep cervical nodes lying in front and behind internal jugular vein; $s$, sternocleidomastoid muscle, retracted so as to show the deep cervical nodes in the upper half of the neck; I, J, internal jugular vein; $O$, omohyoid muscle; $B$, anterior belly of digastric.

The flaps are retracted and all of the following tissues removed in submental region and the submaxillary triangles on both sides of the neck: subcutaneous fat, submaxillary and submental lymph nodes, submaxillary salivary glands and all veins, large or small.

In cases of cancer of the tongue the removal of lymphatic nodes, etc., should not only include the contents of the submaxillary region, but the additional incisions ( $G, C, D$ and $F, B, E$ ), shown in Figures 5, 6, and 7 should be made. These permit free exposure of the lymph nodes lying along the internal and anterior jugular veins beneath the sternocleidomastoid muscles on both sides of the neck and many lymph nodes between the sternocleidomastoid muscle and the muscles of the larynx and at the bifurcation of the common carotid.

If the sternomastoid or the omohyoid be involved, they should be removed, since their extirpation does not interfere greatly with the movements of the head. Similarly if the nodes are adherent to the internal jugular it is best to resect a portion of this vessel. No operation should be considered complete without a thorough removal (through the incisions made over the middle of the sternocleidomastoid muscle) of the deep cervical nodes along the internal jugular. The exposure of the supraclavicular fosse on each side through an incision parallel to the upper portion of the clavicle (Fig. 6) permits an inspection of the lymphatics in this region and removal of the abundant fatty tissue and infected lymphatics. In cases of cancer of the tongue there are two other places in which search should always be made for infected lymph nodes. Thesc are on and within the parotid capsule.

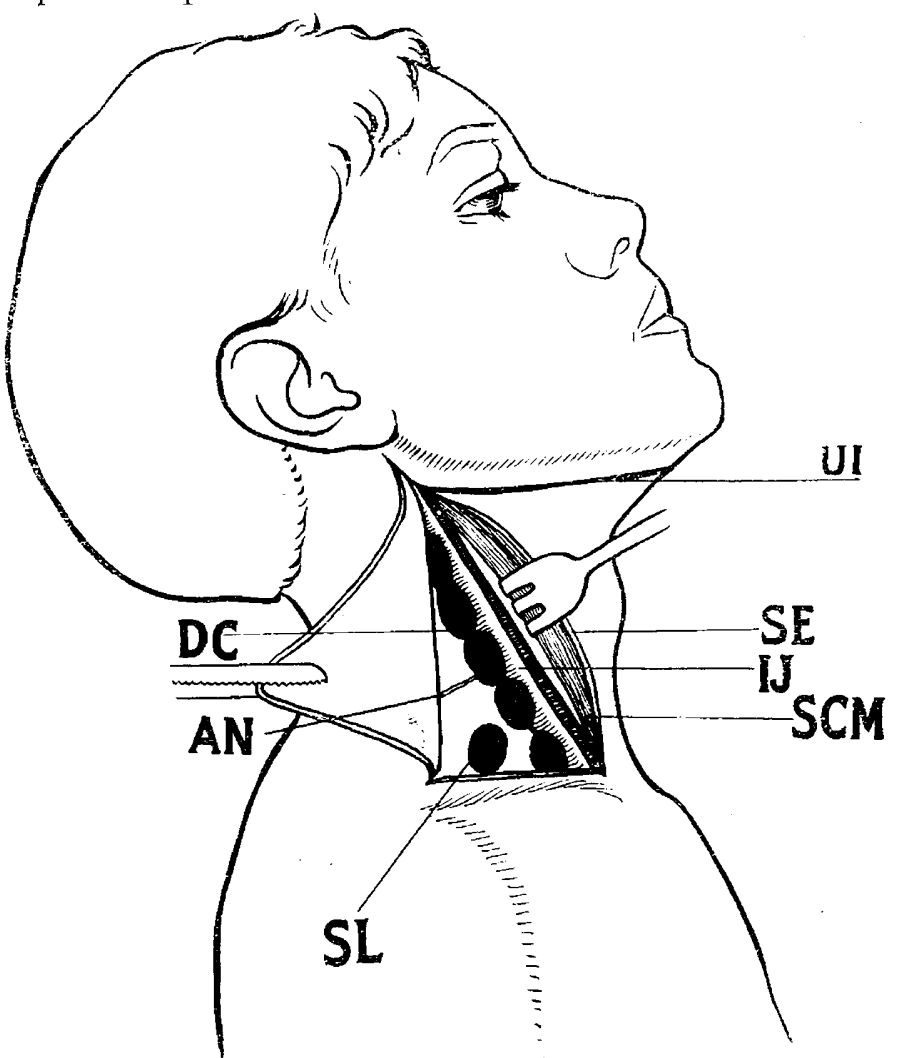

lig. 7.-Method of removing lymph nodes in posterior triangle of neck, and those adherent to posterior aspect of internal jugular vein without resection of the stemocieidomastoid muscle: U, I, incision through which the submaxillary and submental glands have been removed; S, F, skin edge ; I, J, internal jugular vein ; $\mathbf{S}, \mathbf{C}, \mathbf{M}$, sternocleidomastold muscle pulled iorward; S, L, supraclavicular nodes; A, N, spinal accessory nerve; D, C, deep cervical of internal jugular nodes.

3. Hemostasis.-Hemostasis in operations for extirpation of cancer of the lips and of the lymphatic nodes in the submaxillary region is a much simpler problem than in the case of an operation for cancer of the tongue. In the latter the question depends again on whether the organ is removed through the mouth or from below by Kocher's method. In the latter case the incisions described above for the removal of lymphatics greatly facilitate the ligation of the lingual or even of the external carotid arteries. There is, of course, some danger of cerebral embolism following the ligation of 
the external or common carotid. Mortality from this cause is estimated by Crile usually at from 1 to 2 per cent., but at the time of life at which cancer is likely to develop the danger of embolism from this cause increases to from 20 to 30 per cent. A ligation of the lingual arteries through the neck incision before removing the tongue is especially to be recommended as greatly decreasing the amount of hemorrhage and not being followed by cmbolism. In general it may be said that every vessel should be caught with artery forceps and ligated lest they reopen after the clots become dislodged.

Every precaution should be taken to avoid infecting the field of operation either from the primary growth or through careless removal of carcinomatous lymph nodes. 'The primary focus should be cauterized, if possible, and great care taken not to open the lymph nodes during their removal.

4. Mode of Anesthesia, Posture, Etc.-The mode of anesthesia varies accórding to whether or not a preliminary tracheotomy has been performed. If the latter has been done, the anesthetic is given through the canula in the trachea. If no tracheotomy has been performed one can use the method of Crile, viz., inserting a rubber tube through each nostril extending to the level of the epiglottis, or the anesthetic can be administered through the nostrils after being poured on a sponge or pumped through a rubber tube inserted just beyond the external nares with the aid of the modified Brophy apparatus. Chloroform should be given preference on account of the greatly increased amount of salivary secretion after the use of ether. The hypodermic administration of $1 / 4 \mathrm{gr}$. morphin 15 minutes before beginning the operation greatly decreases the amount of anesthetic used.

The best position for thorough removal of the tongue with removal of the lymphatics is with a sand bag under the shoulders and the head thrown well back. Whitehead prefers a sitting posture. In operation on the lips the primary growth can be excised with the head (levated, and then the latter can be lowered to permit removal of the lymph nodes.

\section{TECHNIC IN ADVANCED CASES.}

This has been so well described by Crile in a number of recent articles that it will be necessary to refer only briefly to it here.

In these advanced cases Crile closes the common carotids temporarily by means of a clamp and then proceeds to remove from below upward all of the soft tissues, including the lymph nodes themselves, the sternoeleidomastoid muscles, the internal jugular vein, fat, cte. It is remarkable how little general or local disturbance such radical procedures cause. Whitehead mentions having excised portions of the common carotids in two cases.

SIIAI TIIE OPERATION BE PERFORMED IN ONE OR TWO SITTINGS?

The question arises in these radical operations whether both the primary growth and the lymphatics shall be removed at one sitting. The answer is that it depends, $a$, on the general condition of the patient; $b$, on the extent of involvement; $c$, on the skill and experience of the operator in this class of cases.

In cancer of the lips both opcrations can usually be performed at one sitting. In cancer of the tongue there should be only one sitting unless the patient's condition is such as to permit of an operation requiring in the most experienced hands from 1 to $1 \frac{1}{2}$ hours. The shock from these operations is, as a rule, very slight, so that, if possible, both had better be performed at the same time.

Boyd and Unwin ${ }^{9}$ have called attention to a very important fact, viz., that if the tongue growth is removed at the first sitting and then the lymphatics taken out 1 to 2 weeks later an increased lymph stream follows and the tumor cells are more widely diffused. They advise, if the operation is to be performed in two steps, to remove the glands first and the tongue a week later.

It may be said in general that, with the exception of very old or weak patients, there are many advantages in performing both operations at one sitting, especially if the Kocher method of removing the tongue be employed. Butlin $^{4}$ performs the operation on the tongue first. The removal of lymphatics is done as soon as the patient is able to take food by mouth, i. e., liquid food enough to enable him to fight through the second operation.

My own method has been to perform both operations for cancer of the lips, face and ears at the same sitting. In cancer of the tongue $I$ have carried out both at the same sitting in fairly robust patients without much glandular involvement. If the latter is extensive it is better to follow the plan of Butlin just mentioned.

103 State Street.

\section{THE RADICAL CURE OF ANEURISM.}

PRESENT STATUS OF THE METHOD OF INTRASACCELAR SUTURE, OR ENDOANEURISMORRHAPHY.*

RUDOLPH MATAS, M.D.

Professor of Surgery, Medical Department Tulane Unlversity, Louisiana.

NEW ORLEANS.

SYNOPSIS.

'The term, endoaneurismorraphy, meaning the closure of an aneurismal orifice connecting the trunk of an artery with the aneurismal sac by suturing the orifice from with in the sac and the obliteration of the sac by suture, I. introduced in 1902 , in a paper read before the American Surgical Association at its meeting in Albany, to describe an operation which it was my fortune to apply for the first time at the Charity Hospital of New Orleans while attempting the extirpation of a traumatic brachial aneurism in a negro, March 30,1888 , eighteen years ago. It was after the failure to cure this traumatic aneurism by the application of a proximal and distal ligature of the brachial artery that I attempted the extirpation of the sac; but the extirpation had to be abandoned because of the danger of injuring the median and ulnar nerves which were incorporated in the walls of the sac. The persistence of bleeding, after the removal of the tourniquet, from numerous collaterals which opened into the main trunk at each end of the sac, compelled me to adopt the suture with needle and silk to occlude the bleeding orifices. This simple expedient, suggested by the urgent hemostatic necessities of the case, appeared to be so easy and satisfactory that it seemed to me that any surgeon similarly situated, would have instinctively adopted this way of extricating himself from what would otherwise have been a difficult situation.

The detailed report of this case ${ }^{1}$ attracted no notice,

* Read in the Section on Surgery and Anatomy of the American Iedical Association, at the Fiftr-seventh Annual Sesslon, June, 1906.

1. Medica! News, Thilarlelphia. Net. 27. 18R8. 